\title{
THE SUBSTITUTION OF REGULATED BROMINATED FLAME RETARDANTS IN PLASTIC PRODUCTS AND WASTE AND THE DECLARED PROPERTIES OF THE SUBSTITUTES IN REACH
}

\section{Pierre Hennebert *}

Ineris (French National Institute for Industrial Environment and Risks), BP 2, F-60550 Verneuil-en-Halatte, France

Article Info:

Received:

26 February 2021

Revised:

9 July 2021

Accepted:

23 August 2021

Available online:

30 September 2021

Keywords:

Total bromine

Brominated flame retardants

Substitution

Classification

Registration

\begin{abstract}
Plastics containing brominated flame retardants (BFR) currently contain both "legacy" regulated and non-regulated BFR (R-BFRs and NR-BFRs), as evidenced by the increasingly lower correspondence over time between total bromine and R-BFRs content. The portion of substitutive NR-BFR present in the plastics and their toxicity and ecotoxicity properties are documented. Data relating to plastics and foam present in electrical and electronic equipment (EEE), waste EEE, vehicles, textiles and upholstery, toys, leisure and sports equipment show how $88 \%$ of plastic waste contains bromine from NR-BFRs. BFR substances mentioned in the catalogs of the three main producers (Albemarle, ICL, Lanxess) and BFR on the official used list of 418 plastic additives in the EU were gathered and the toxic and ecotoxic properties of these compounds as listed in their ECHA registration dossier were compiled. Fifty-five preparations using 34 NR-BFRs substances, including polymers and blends, were found. Seventeen of these substances featured an incomplete dossier, 12 were equipped with a complete dossier, whilst 11 substances (including 2 ill-defined blends) should be reassessed. Eight substances have been notified for assessment by the ECHA as persistent, bioaccumulative and toxic, or as endocrine disruptors, including decabromodiphenylethane; 3 substances display functional concentrations (the concentration of additives that retards flame) exceeding the concentration limits classifying a waste as hazardous but are "reactive" (they bind to the polymer). The technical limit of $2000 \mathrm{mg}$ total $\mathrm{Br} / \mathrm{kg}$ indicated for further recycling (EN 50625-3-1) relates to all brominated substances and is relevant in the sorting of all poorly classified new substances.
\end{abstract}

\section{INTRODUCTION}

Some plastic constituents of products include flame retardant compounds to protect the users against fire: electrical and electronic equipment, automotive and transport vehicles, construction piping and insulating material, furniture foams and upholstery, professional textiles, and many others. They are used in functional concentrations ranging from 3 to $15 \% \mathrm{w} / \mathrm{w}$, with up to $30 \%$ tetrabromobisphenol $\mathrm{A}$ in epoxy resins (Haarman et al. 2020). Animals and people are exposed. For instance, Brominated flame retardants (BFRs), including polybrominated diphenyl ethers (PBDEs), 1,2-bis (2,4,6-tribromophenoxy) ethane (BTBPE), decabromodiphenyl ethane (DBDPE), and polybrominated biphenyls (PBBs) were found in children's toys purchased from South China, but it accounts for a small proportion of their daily BFR exposure (Cheng SJ et al. 2009). Lower use of flame retardants with the same fire protection are considered (Charbonnet et al. 2020). While many of the toxic FRs have been eliminated and replaced by other FRs, existing products containing toxic or potentially toxic chemical FRs will remain in use for decades, and new products containing these and similar chemicals will permeate the environment. When such products reach the end of their useful life, proper disposal methods are needed to avoid health and ecological risks (Lucas et al., 2018).

Low concentrations of bromine and (regulated $\mathrm{R}$ - or non-regulated NR-) brominated flame retardants (ineffective for flame retardancy) are also found in plastics due to improper recycling, showing that a circular economy must be controlled to avoid looping unwanted substances (Hennebert 2020).

Brominated flame retardants (BFRs) are numerous. So far, the regulated BFRs (R-BFRs), according to the Regulations 2004/850/EC on Persistent Organic Pollutants (POPs) and 2008/98/EC, are:

\section{- Polybromobiphenyls (PBBs - hexabromobiphenyl 50}


$\mathrm{mg} / \mathrm{kg}$ );

- Polybromodiphenylethers (PBDEs - mainly decabromodiphenylether decaBDE - sum 4-, 5-, 6-, 7-, 10-BDE $1000 \mathrm{mg} / \mathrm{kg}$ );

- Hexabromocyclododecane (HBCDD $1000 \mathrm{mg} / \mathrm{kg}$ ) and Tetrabromobisphenol A (TBBPA $2500 \mathrm{mg} / \mathrm{kg}$ ).

Currently, PBBs are no longer found in plastic waste. For some years, PBDEs were largely replaced by decabromodiphenylethane: the substitution is now complete since the inclusion of decaBDE in the POPs list in 2019. DecaBDE still has exemption in the Stockholm Convention including certain EEE and is still produced and used. TBBPA is used in polyester and polycarbonate resins, typically for electrical and electronic equipment, and HBCDD was used up to 2014 in Europe but is still produced in China and is found only in expanded or extruded polystyrene. Most of HBCD containing insulation used in construction the last 40 years is still in use and will end the next 50 or even 100 years in construction and demolition waste polymers.

The recycling of the plastics must start with a separation of the plastic pieces/shreds with these R-BFRs that have a concentration above the concentration limit. Sorting of these plastics is performed by surrogate methods: atomic density (by X-ray transmission) or solid density (by flotation). A technical specification of the European standardization committee set the limit of $2,000 \mathrm{mg} \mathrm{Br} /$ $\mathrm{kg}$ as the maximum content for recycling (EN 50625-3-1). If legacy additives are removed and destructed safely, a recycling technology provides a method to manage such waste streams in an environmentally friendly and economically feasible manner. Therefore, it is important that either recyclers commit and adopt the concept of decontamination, by sorting or solvent based technologies (Wagner and Schlummer 2020).

Brominated plastics now contain both "legacy" regulated and non-regulated BFRs, as is evidenced by the increasingly lower correspondence, with time, between total bromine and R-BFRs content (Haarman et al. 2020). Therefore the question arise to what extent is the part of the substitutive BFR in plastics? And what are the toxicity and ecotoxicity properties of these substitutes? As substitution is going faster than classification and regulation, and as substances with similar structure are used (for instance decabromodiphenylethane replacing decabromodiphenylether), all these new BFRs should be investigated and their properties assessed and published to avoid regrettable substitutes (Fantke et al. 2015). This paper investigates the substitutes' declared chemical properties on human health and the environment in ECHA registration dossier.

\section{MATERIAL AND METHODS}

\subsection{Assessment on R-BFRs and NR-BFRs in WEEE plastic}

The substituting NR-BFRs are not measured during routine quality control. Nevertheless, they can be assessed by the difference between total bromine and the bromine from regulated BFRs (R-BFRs), which are routinely measured. Sample preparation of plastic shreds is done according to
EN 15002. Total bromine is measured by ionic chromatography after combustion in an oxygen bomb. The R-BFRs are measure according to EN 62321-6.

Data from plastics and foam for electrical and electronic equipment (EEE), EEE waste, vehicles, textiles and upholstery, toys, leisure and sports equipment have been gathered, with detailed various origin (Hennebert 2020). Probably, the oldest wastes are cathode ray tubes (CRT), produced about 30 years ago. Some samples are individual particles or articles, and others are composite samples with or without "enough" particles to be representative of the waste lot to be characterized (Hennebert 2019, Hennebert and Beggio 2021, Beggio and Hennebert 2021). Here, the representativity of the laboratory sample is not a challenge, since only paired data (R-BFRs and $\mathrm{Br}$ ) have been used, measured on the same particle/article or laboratory sample (after pre-treatment). The mean stoichiometric $\mathrm{Br}$ concentration in the different R-BFRs is $76 \% \mathrm{w} / \mathrm{w}$. DecaBDE, by far the most present R-BFRs, has a mean $\mathrm{Br}$ concentration of $83 \% \mathrm{w} / \mathrm{W}$. The corresponding reverse ratios R-BFRs/Br are 1.31 and 1.20, respectively.

2.2 Approach to compile the list of BFRs and source information on REACh properties

The BFR substances mentioned in the catalogs of the three main producers in industrial countries (Albemarle, ICL, Lanxess) were listed and their declared chemical properties on human health and the environment, as stated in their European ECHA registration dossier (https://echa. europa.eu/information-on-chemicals), were gathered. The large production in China ( $>50 \%$ of all BFRs) has not been documented. The 9 brominated flame retardants from the list of the 418 "Plastic Additives Initiative" (a common list by ECHA and industry of additives used in quantity more than 100 tons per year in the EU) presently used in the EU (https://echa.europa.eu/mapping-exercise-plastic-additives-initiative\#table) have also been used.

\section{RESULTS AND DISCUSSION}

\subsection{Monitoring of the share of R-BFR and NR-BFRs in WEEE plastic}

For clarity, all the measured R-BFRs are summed. As explained, in practice, $\mathrm{PBBs}$ are not found, decaBDE is dominant in the PBDEs ( $>90 \%$ R-BFRs), HBCDD is present in expanded EPS (and not mixed with the other R-BFRs), and TBBPA is sometimes present. In these data, not all the R-BFRs were measured for all the samples, but PBDEs and especially decaBDE have always been measured. Total bromine has been measured by XRF for particles or articles, or after shredding, combustion in an oxygen bomb and ionic chromatography for the composite samples. R-BFRs have been measured by standardized chromatographic methods (Figure 1, Table 1).

The stoichiometric ratio $\mathrm{R}-\mathrm{BFRs} / \mathrm{Br}$ is 1.31 for all R-BFRs, and for decaBDE it is 1.20 . Considering sampling and analytical variability, this range can be extended $[1 ; 1.5]$ (vertical lines in Figure 2). Only $8 \%$ of the plastics have a concentration of R-BFRs between 1 and 1.5 times the concentration of bromine in this set of data. $88 \%$ of plastic waste contains bromine from $\operatorname{BFR}(\mathrm{s})$ other than the reg- 
All data $(n=286)$

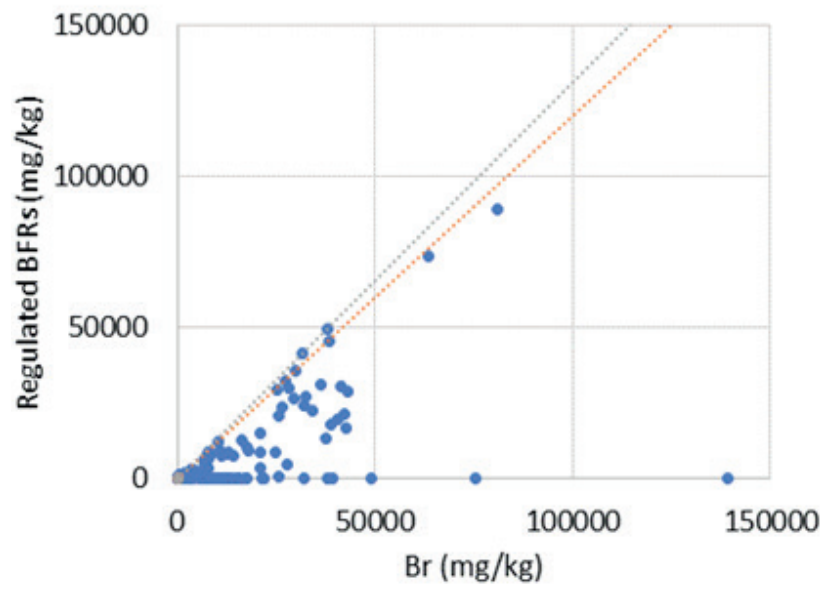

$\mathrm{Br}<5000 \mathrm{mg} / \mathrm{kg}(\mathrm{n}=203)$

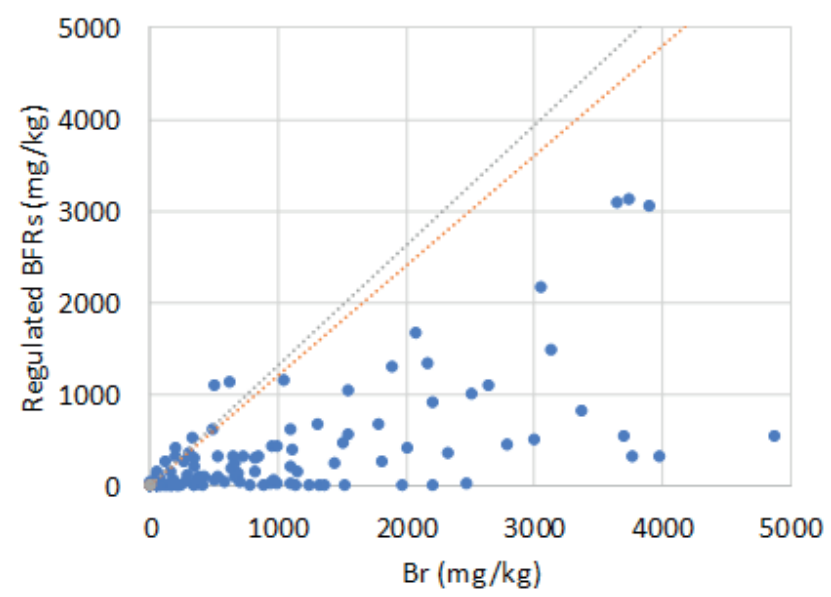

FIGURE 1: Regulated brominated flame retardants (R-BFRs) as a function of total bromine content (left) and the same data with $\mathrm{Br} \leq 5,000$ $\mathrm{mg} / \mathrm{kg}$ (right), with stoichiometric ratio $\mathrm{R}-\mathrm{BFRs} / \mathrm{Br}$ of $1.20 \mathrm{w} / \mathrm{w}$ (orange line) and $1.31 \mathrm{w} / \mathrm{w}$ (grey line).

ulated ones. Only $4 \%$ of the data have a concentration of R-BFRs greater than 1.5 times the concentration of $\mathrm{Br}$ (with maximum R-BFRs $=1135 \mathrm{mg} / \mathrm{kg}$ ). This discrepancy is due mainly to sample preparation (i.e. the test portions of 1 gram that is used for $\mathrm{Br}$ and for R-BFRs measurement do not have the same composition), and secondarily to analytical variability (CVanalysis of R-BFRs $=13 \%$, Hennebert 2019).

\subsection{REACh evaluation status and hazard information of NR-BFRs substitutes}

55 preparations using 34 BFR substances (other than the regulated ones) can be found in the catalogs of the three main producers, consulted in November 2019, and in the list of 418 additives. A summary is presented in Table 2 and Figure 3 and will be discussed first. Detailed results per substance are presented in Table 3.

The 2 R-BFRs and 7 NR-BFRs declared in the list of 418 additives actually used in the EU is presented in Table 4. It is not explained on the ECHA site why the two R-BFRs HBCDD and DecaBDE are included. The initiative started in 2016 and the excel file was published in 2019. The 7 NR-BFRs are included in Group 3 or Group 4 of the list established from the producer's catalog (Table 3 ). The correspondence is presented in the second column of Table 4.

Some substances (frequently proprietary) have no CAS number and hence no dossier can be found in the ECHA site (called here Group 1). Three of these substances are declared as not registered for sale in Europe. Another group of substances has a CAS number but has not been declared to ECHA and has no dossier (Group 2). Another group of substances has a CAS number and a dossier, but no hazard statement codes (HSC) (Group 3). Most of their registration dossier items are just filled in as "information non available - n.a.". A fourth group of substances has a CAS number, a more complete dossier, and a HSC (Group 4). The groups are the following (Table 3):

- Group 1: 10 substances have no CAS number and no dossier in ECHA, among which 3 substances are not registered for sale in Europe and thus logically without

RBFRs / $\mathrm{Br}<1$ for $88 \%$ of the data $(n=216)$

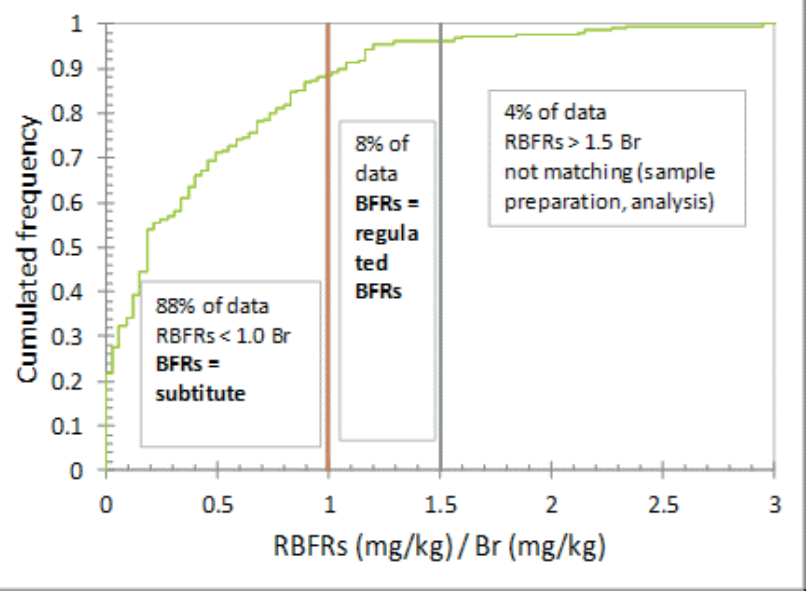

FIGURE 2: Distribution of individual R-BFRs/Br ratio (only data > LOQ) with practical $\mathrm{R}-\mathrm{BFRs} / \mathrm{Br}$ ratio including sampling and an analysis variability of 1 (orange) and 1.5 (grey)..

TABLE 1: Distributions of $\mathrm{Br}, \mathrm{R}-\mathrm{BFRs}$ and $\mathrm{R}-\mathrm{BFRs} / \mathrm{Br}$ ratio (CV coefficient of variation = relative standard deviation RSD = standard deviation/mean).

\begin{tabular}{|c|c|c|c|c|c|c|c|c|}
\hline Parameter & Unit & $\mathbf{n}$ & Minimum & Median & Mean & Maximum & $\begin{array}{l}\text { Standard } \\
\text { deviation }\end{array}$ & CV \\
\hline $\mathrm{Br}$ & $\mathrm{mg} / \mathrm{kg}$ & 286 & $<10$ & 596 & 7196 & 139000 & 14962 & 2.08 \\
\hline Sum R-FBRs & $\mathrm{mg} / \mathrm{kg}$ & 286 & $<10$ & 18 & 3483 & 89200 & 10258 & 2.95 \\
\hline R-BFRs / Br & - & 216 & 0.00 & 0.20 & 0.41 & 2.97 & 0.50 & 1.21 \\
\hline
\end{tabular}


TABLE 2: Summary of the classification of substitutes of regulated brominated flame retardants (R-BFRs) in the REACh registration dossiers (PBT list $=$ PBT evaluation list, ED list $=$ ED evaluation list).

\begin{tabular}{|c|c|c|c|c|c|c|c|c|c|}
\hline $\begin{array}{l}\text { Preparations and } \\
\text { substances }\end{array}$ & $\mathbf{n}$ & CAS \# & $\mathbf{n}$ & $\begin{array}{l}\text { Classification (by } \\
\text { notifiers) }\end{array}$ & $\mathbf{n}$ & $\begin{array}{l}\text { Classified for HP of waste } \\
\text { ( } C L=\text { concentration limit } \\
\text { for hazardous waste) }\end{array}$ & $\begin{array}{l}\text { Notifica- } \\
\text { tions (by } \\
\text { ECHA) }\end{array}$ & $\mathbf{n}$ & $\begin{array}{l}\text { Group name } \\
\text { in this paper }\end{array}$ \\
\hline $\begin{array}{l}\text { Preparations ( } 3 \\
\text { main producers) }\end{array}$ & 55 & & & & & & & & \\
\hline \multirow[t]{4}{*}{ Substances } & \multirow[t]{4}{*}{34} & No & $10 *$ & & & & & & 1 \\
\hline & & \multirow[t]{3}{*}{ Yes } & \multirow[t]{3}{*}{24} & $\begin{array}{l}\text { No dossier in ECHA } \\
\text { site }\end{array}$ & 2 & & & & 2 \\
\hline & & & & $\begin{array}{l}\text { Dossier in ECHA } \\
\text { site, no Hazard } \\
\text { Statement in the } \\
\text { dossier }\end{array}$ & 10 & & $\begin{array}{l}\text { PBT list, } \\
\text { PBT and ED } \\
\text { list, ED list }\end{array}$ & $3+1+2^{\star \star \star}$ & 3 \\
\hline & & & & Classified with HSC & 12 & 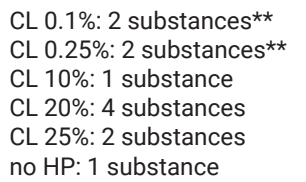 & $\begin{array}{l}\text { PBT list, } \\
\text { PBT list and } \\
\text { ED list }\end{array}$ & 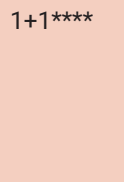 & 4 \\
\hline
\end{tabular}

* Including 3 proprietary substances not registered for sale in Europe

** The 3 substances with a concentration limit for hazardous waste below the functional concentration are a reactive intermediate for high molecular weight flame retardants and will normally not be found as separate substances in plastics but are linked to the polymer matrix: dibromo neopentyl glycol (CAS 36483-57-5), tribromo neopentyl alcohol (same CAS 36483-57-5) for PU foam, and 2,4,6 tribromophenol (CAS 118-79-6) for epoxides, respectively.

*** PBT list: 2,4,6-Tris (2,4,6-tribromophenoxy) -1,3,5 triazine (CAS 25713-60-4); brominated flame retardant, aromatic, combined with an imide structure (CAS 32588-76-4); decabromodiphenyl ethane (CAS 84852-53-9). The PBT and ED list: 1,3-dibromo-5-\{2-[3,5-dibromo-4-(2,3-dibromopropoxy)phenyl]propan-2-yl\}-2-(2,3-dibromopropoxy)benzene (CAS 21850-44-2), ED list: 1,1'-(isopropylidene)bis[3,5-dibromo-4-(2,3-dibromo-2-methylpropoxy)benzene] (CAS 97416-84-7), 2,2-bis(bromomethyl)propane-1,3-diol (CAS 3296-90-0)

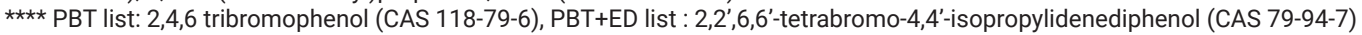

CAS number $=$ Chemical Abstracts Service number

$\mathrm{HP}=$ hazard property of waste

HSC = hazard statement code

PBT list = "under evaluation as persistent, bioaccumulative and toxic" by ECHA

ED list = "being assessed as an endocrine disruptor" by ECHA

a dossier, 4 substances are declared as polymeric or an end-capped polymer (that have not been declared in the REACh system) and one substance is in pellet form (decabromodiphenylethane, the classical substitute

Registration of BFRs other than POP-regulated BFRs (55 preparations, 34 substances)

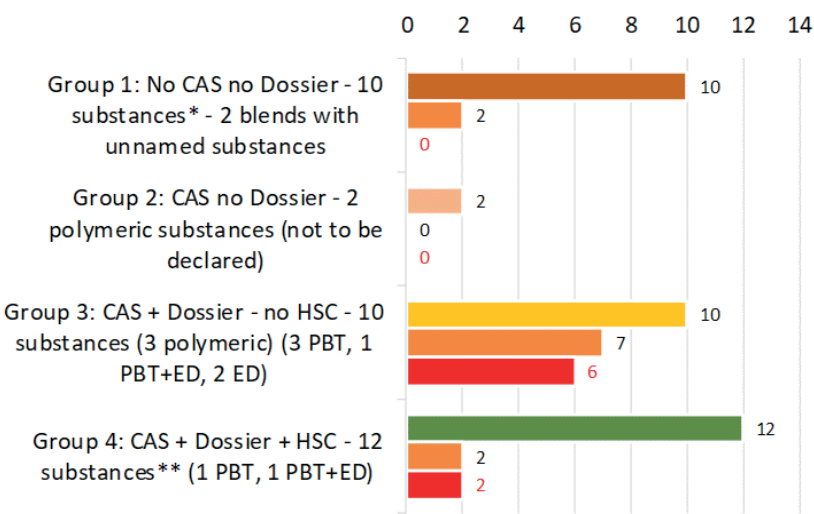

FIGURE 3: Classification of substitutes of regulated brominated flame retardants (R-BFRs) in the REACh registration dossier of ECHA. For each group: first bar: total number of substances; second bar: number of substances to assess or re-assess; third bar: number of substances under assessment by ECHA. (CAS = CAS number, Dossier = dossier in ECHA site, $\mathrm{HSC}=$ presence of hazard statement code of the substance, PBT = "under evaluation as persistent, bioaccumulative and toxic" by ECHA, ED = "being assessed as an endocrine disruptor" by ECHA). Notes: *3 substances not for sale in Europe, 4 polymeric, 1 physical form; ** 3 substances with functional concentration $>$ hazardous concentration for waste. of banned decabromodiphenylether, which has a CAS number and a dossier, and is further detailed in Group 3). The remaining 2 substances are "Blend brominated flame retardant, diol, phosphate ester" and "Blend brominated flame retardant, halogen, phosphorus". Mixtures have not been declared, but their substances have. It is not known if the unnamed individual substances of these blends are registered;

- Group 2: 2 substances that have a CAS number but no dossier. These substances are polymers or building blocks for a polymer, that have not been declared in the REACh system;

- Group 3: 10 substances that have a CAS number and a dossier but without hazard statements and therefore not classified. The ECHA has, however, placed 6 of these substances on the PBT and/or ED list ("under evaluation as persistent, bioaccumulative and toxic" and "being assessed as an endocrine disruptor"). The most common result of these re-evaluations is that the substances are assessed as hazardous, and that the registration dossier must be modified. 3 other substances are polymers or building blocks for a polymer;

- Group 4: 12 substances among which 2 polymers, 2 salts of bromide $\mathrm{Br}$ - (potassium and ammonium), 4 tetrabromo phthalic derivatives, 2 brominated pentyl alcohols ( 5 carbons) and 1 bromophenol (6 carbons). The ECHA has, however, placed 2 of these substances (decabromodiphenylethane: PBT evaluation list; 2,2',6,6'-tetrabromo-4,4'-isopropylidenediphenol: PBT evaluation list + ED evaluation list). 
TABLE 3: Substitutes of regulated brominated flame retardants (R-BFRs) as found in the catalogs of the three main producers, CAS number, formula, and their classification in ECHA REACh registration dossiers by the notifiers, ECHA notifications, presence of a Brief Profile (BP), hazard statement codes, corresponding concentration limit for classification as hazardous waste, lowest concentration limit and hazard property of the lowest concentration limit (HP $4=$ irritant, HP $5=$ toxic, HP 7 = carcinogenic, HP 11 = mutagenic, HP $14=$ ecotoxic). Substances to re-evaluate are red coloured cells.

\begin{tabular}{|c|c|c|c|c|c|c|c|c|c|}
\hline Group and Substances & Name/Note & CAS No & Formula & $\begin{array}{l}\text { ECHA } \\
\text { (Clas- } \\
\text { sifica- } \\
\text { tion) }\end{array}$ & $\begin{array}{l}\text { ECHA } \\
\text { Noti- } \\
\text { fica- } \\
\text { tions }\end{array}$ & BP & $\begin{array}{c}\text { Hazard Statement } \\
\text { Code, Concentration } \\
\text { Limit (Waste Hazard } \\
\text { Property) }\end{array}$ & $\begin{array}{l}\text { Low- } \\
\text { est } \\
\text { CL }\end{array}$ & $\begin{array}{c}\text { HP of } \\
\text { lowest } \\
\text { CL }\end{array}$ \\
\hline
\end{tabular}

GROUP 1 Substances: No CAS (10)

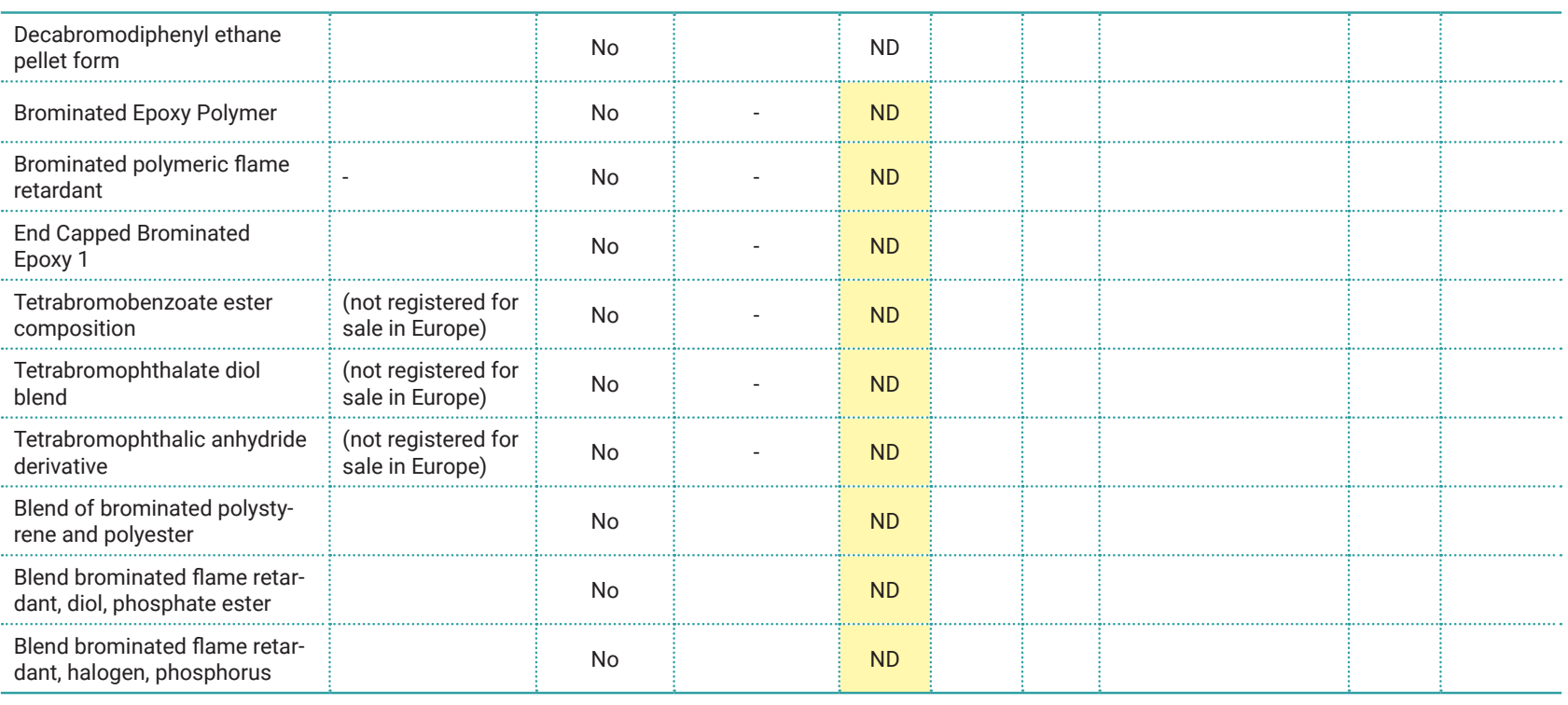

GROUP 2 Substances: No dossier (2)

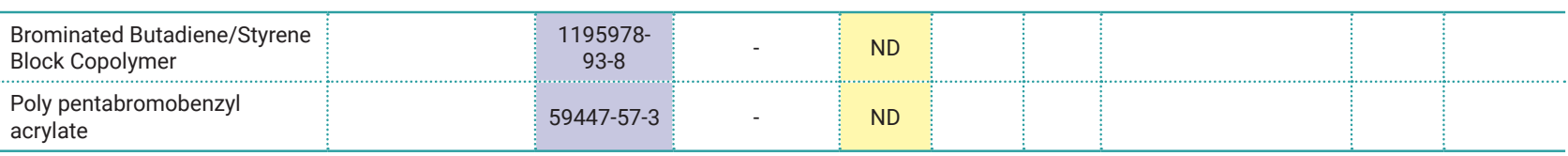

GROUP 3 Substances: No Hazard Statement (10)

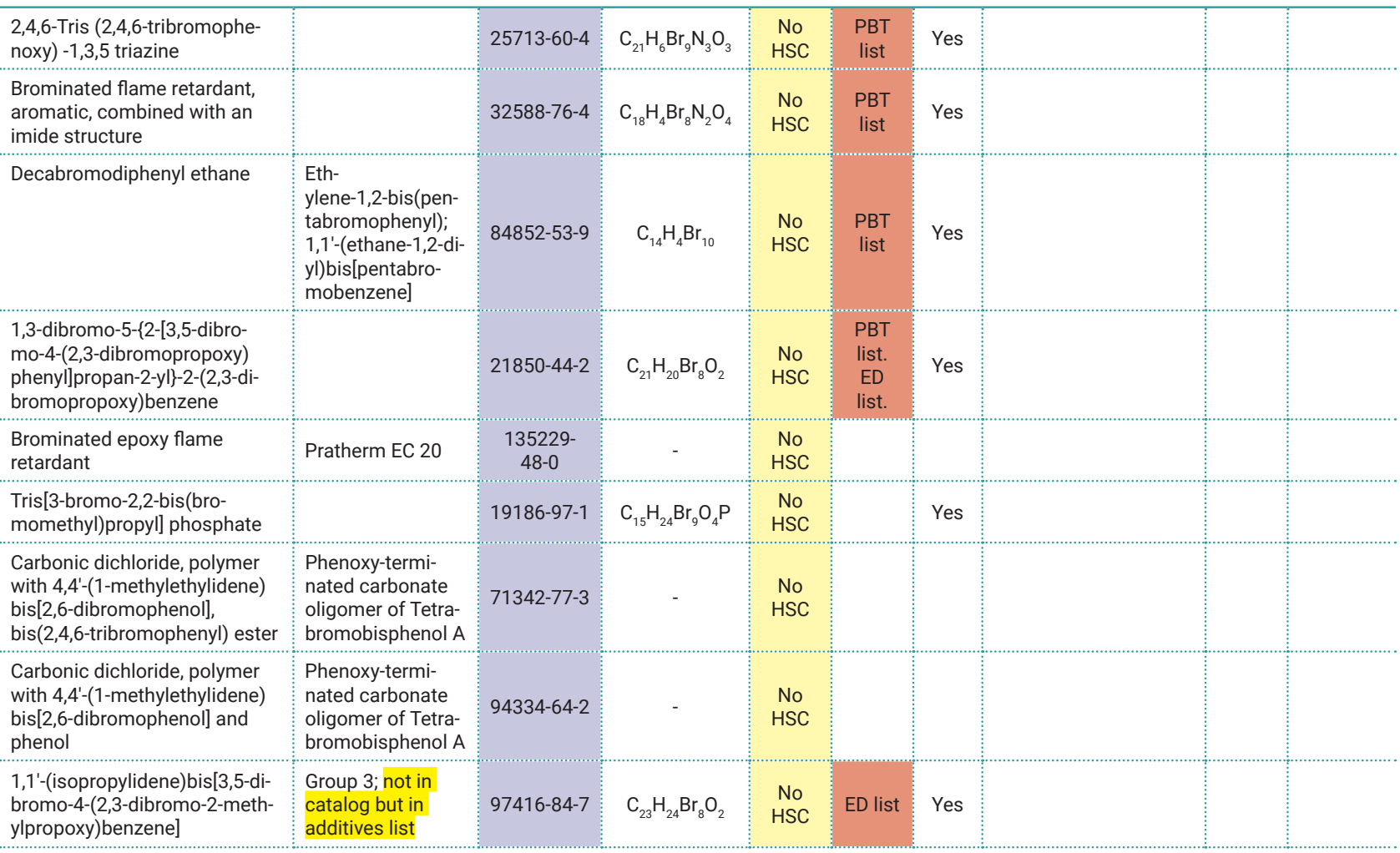




\begin{tabular}{|c|c|c|c|c|c|c|c|c|c|}
\hline Group and Substances & Name/Note & CAS No & Formula & $\begin{array}{l}\text { ECHA } \\
\text { (Clas- } \\
\text { sifica- } \\
\text { tion) }\end{array}$ & $\begin{array}{l}\text { ECHA } \\
\text { Noti- } \\
\text { fica- } \\
\text { tions }\end{array}$ & BP & $\begin{array}{l}\text { Hazard Statement } \\
\text { Code, Concentration } \\
\text { Limit (Waste Hazard } \\
\text { Property) }\end{array}$ & $\begin{array}{l}\text { Low- } \\
\text { est } \\
\text { CL }\end{array}$ & $\begin{array}{l}\text { HP of } \\
\text { lowest } \\
\text { CL }\end{array}$ \\
\hline $\begin{array}{l}\text { 2,2-bis(bromomethyl)pro- } \\
\text { pane-1,3-diol }\end{array}$ & $\begin{array}{l}\text { Group 3; not in } \\
\text { catalog but in } \\
\text { additives list }\end{array}$ & $3296-90-0$ & $\mathrm{C}_{23} \mathrm{H}_{24} \mathrm{Br}_{8} \mathrm{O}_{2}$ & $\begin{array}{l}\text { No } \\
\text { HSC }\end{array}$ & ED list & Yes & & & \\
\hline \multicolumn{10}{|c|}{ GROUP 4 Substances: with Hazard Statement Code(s) (12) } \\
\hline Dibromo neopentyl glycol & & $36483-57-5$ & $\mathrm{C}_{5} \mathrm{H}_{10} \mathrm{Br}_{2} \mathrm{O}_{2}$ & $\begin{array}{c}\text { H302 } \\
\text { H315 } \\
\text { H319 } \\
\text { H335 } \\
\text { H340 } \\
\text { 1B } \\
\text { H341 } \\
\text { H350 } \\
\text { 1B } \\
\text { H351 } \\
\text { H373 } \\
\text { H413 }\end{array}$ & & Yes & $\begin{array}{c}\text { H302 } 25 \% \text { (HP 6) } \\
\text { H315 H319 20\% (HP 4) } \\
\text { H335 20\% (HP 5) } \\
\text { H340 0.1\% (HP 11) } \\
\text { H341 1\% (HP 11) } \\
\text { H350 0.1\% (HP 7) } \\
\text { H351 1\% (HP 7) } \\
\text { H373 - (no HP) } \\
\text { H413 25\% (HP 14) }\end{array}$ & $0.10 \%$ & $\begin{array}{c}\text { HP } 7 \text { HP } \\
11\end{array}$ \\
\hline Tribromo neopentyl alcohol & $\begin{array}{l}\text { 2,2-dimethylpro- } \\
\text { pan-1-ol, tribromo } \\
\text { derivative }\end{array}$ & $36483-57-5$ & $\mathrm{C}_{5} \mathrm{H}_{9} \mathrm{Br}_{3} \mathrm{O}$ & $\begin{array}{c}\mathrm{H} 302 \\
\mathrm{H} 319 \\
\mathrm{H} 340 \\
1 \mathrm{~B} \\
\mathrm{H} 341 \\
\mathrm{H} 350 \\
\text { 1B } \\
\mathrm{H} 412\end{array}$ & & Yes & $\begin{array}{c}\text { H302 25\% (HP 6) } \\
\text { H315 H319 20\% (HP 4) } \\
\text { H340 0.1\% (HP 11) } \\
\text { H341 1\% (HP 11) } \\
\text { H350 0.1\% (HP 7) } \\
\text { H412 25\% (HP 14) }\end{array}$ & $0.10 \%$ & $\begin{array}{l}\text { HP } 7 \text { HP } \\
11\end{array}$ \\
\hline 2,4,6-tribromophenol & & $118-79-6$ & $\mathrm{C}_{6} \mathrm{H}_{3} \mathrm{Br}_{3} \mathrm{O}$ & $\begin{array}{l}\text { H301 } \\
\text { H302 } \\
\text { H312 } \\
\text { H315 } \\
\text { H317 } \\
\text { H319 } \\
\text { H332 } \\
\text { H335 } \\
\text { H400 } \\
\text { H410 } \\
\text { H411 }\end{array}$ & $\begin{array}{l}\text { PBT } \\
\text { list }\end{array}$ & Yes & $\begin{array}{c}\text { H301 5\% (HP 6) } \\
\text { H302 25\% (HP 6) } \\
\text { H312 55\% (HP 6) } \\
\text { H315 H319 20\% (HP 4) } \\
\text { H332 22.5\% (HP 6) } \\
\text { H335 20\% (HP 5) } \\
\text { H400 25\% (HP 14) } \\
\text { H410 0.25\% (HP 14) } \\
\text { H411 25\% (HP 14) }\end{array}$ & $0.25 \%$ & $\begin{array}{l}\mathrm{HP} 14 \\
(\mathrm{H} 410)\end{array}$ \\
\hline Potassium bromide & & $7758-02-3$ & BrK & $\begin{array}{l}\text { H315 } \\
\text { H318 } \\
\text { H319 } \\
\text { H335 } \\
\text { H336 } \\
\text { H373 } \\
\text { H412 }\end{array}$ & & Yes & $\begin{array}{c}\text { H315+H319 20\% } \\
\text { (HP 4) } \\
\text { H318 10\% (HP 4) } \\
\text { H335 20\% (HP 5) } \\
\text { H336 - (no HP) } \\
\text { H373 10\% (HP 5) } \\
\text { H412 25\% (HP 14) }\end{array}$ & $10 \%$ & $\begin{array}{l}\text { HP } 4 \\
\text { HP } 5\end{array}$ \\
\hline Ammonium bromide & & $12124-97-9$ & $\mathrm{BrH}_{4} \mathrm{~N}$ & $\begin{array}{l}\text { H315 } \\
\text { H319 } \\
\text { H335 }\end{array}$ & & Yes & $\begin{array}{c}\text { H315+H319 20\% } \\
(\text { HP 4) } \\
\text { H335 20\% (HP 5) }\end{array}$ & $20 \%$ & $\begin{array}{l}\text { HP } 4 \\
\text { HP } 5\end{array}$ \\
\hline $\begin{array}{l}\text { Bis(2-ethylhexyl) tetrabromoph- } \\
\text { thalate }\end{array}$ & $\begin{array}{l}\text { Tetrabromophthal- } \\
\text { ate ester }\end{array}$ & $26040-51-7$ & $\mathrm{C}_{24} \mathrm{H}_{34} \mathrm{Br}_{4} \mathrm{O}_{4}$ & H319 & & & $\begin{array}{c}\mathrm{H} 315+\mathrm{H} 31920 \% \\
\text { (HP 4) }\end{array}$ & $20 \%$ & HP 4 \\
\hline Brominated Epoxy Oligomery & $\begin{array}{l}\text { Tetrabromobisphe- } \\
\text { nol A diglycidyl } \\
\text { ether, Brominated } \\
\text { Epoxy Polymer }\end{array}$ & 68928-70-1 & - & $\begin{array}{l}\mathrm{H} 315 \\
\mathrm{H} 319\end{array}$ & & & $\begin{array}{c}\mathrm{H} 315+\mathrm{H} 31920 \% \\
\text { (HP 4) }\end{array}$ & $20 \%$ & HP 4 \\
\hline $\begin{array}{l}\text { Brominated Polystyrene, acry- } \\
\text { late copolymer }\end{array}$ & $\begin{array}{l}\text { Benzene, ethe- } \\
\text { nyl-,homopolymer, } \\
\text { brominated }\end{array}$ & $88497-56-7$ & - & H319 & & & $\begin{array}{c}\text { H315+H319 20\% } \\
\text { (HP 4) }\end{array}$ & $20 \%$ & HP 4 \\
\hline $\begin{array}{l}\text { 2-(2-hydroxyethoxy)ethyl } \\
\text { 2-hydroxypropyl 3,4,5,6-tetrabro- } \\
\text { mophthalate }\end{array}$ & $\begin{array}{l}\text { Tetrabromophthal- } \\
\text { ate diol, Tetrabro- } \\
\text { mophthalate diol }\end{array}$ & 20566-35-2 & $\mathrm{C}_{15} \mathrm{H}_{16} \mathrm{Br}_{4} \mathrm{O}_{7}$ & $\mathrm{H} 412$ & & Yes & H412 25\% (HP 14) & $25 \%$ & $\begin{array}{l}\mathrm{HP} 14 \\
(\mathrm{H} 412)\end{array}$ \\
\hline $\begin{array}{l}\text { Reaction products of tetra- } \\
\text { bromophthalic anhydride } \\
\text { with 2,2'-oxydiethanol and } \\
\text { methyloxirane }\end{array}$ & & 77098-07-8 & - & $\mathrm{H} 412$ & & Yes & H412 25\% (HP 14) & $25 \%$ & $\begin{array}{l}\mathrm{HP} 14 \\
(\mathrm{H} 412)\end{array}$ \\
\hline Tetrabromophthalic anhydride & & $632-79-1$ & $\mathrm{C}_{8} \mathrm{Br}_{4} \mathrm{O}_{3}$ & H317 & & Yes & H317 - (no HP) & no $C L$ & no HP \\
\hline $\begin{array}{l}\text { 2,2',6,6'-tetrabromo-4,4'-isopro- } \\
\text { pylidenediphenol }\end{array}$ & $\begin{array}{l}\text { Group 4; not in } \\
\text { catalog }\end{array}$ & $79-94-7$ & $\mathrm{C} 15 \mathrm{H} 12 \mathrm{Br} 4 \mathrm{O} 2$ & $\begin{array}{l}\mathrm{H} 400 \\
\mathrm{H} 410\end{array}$ & $\begin{array}{l}\text { PBT } \\
\text { list. ED } \\
\text { Ilist. } \\
\text { ED }\end{array}$ & Yes & $\begin{array}{l}\mathrm{H} 400 \mathrm{25} \% \text { (HP 14) } \\
\mathrm{H} 4100.25 \%(\mathrm{HP} 14)\end{array}$ & $0.25 \%$ & $\begin{array}{l}\mathrm{HP} 14 \\
(\mathrm{H} 410)\end{array}$ \\
\hline $\begin{array}{l}\text { BP = Brief Profile on ECHA site (a } \\
E D \text { list = "Under assessment as Er } \\
H S C=\text { hazard statement code } \\
N D=\text { no dossier } \\
\text { PBT list = "Under assessment as } \\
\text { (HP } 14 \text { calculated without multipl) }\end{array}$ & $\begin{array}{l}\text { summary document or } \\
\text { docrine Disrupting" } \\
\text { ersistent, Bioaccumul } \\
\text { ing "M-factors") }\end{array}$ & tive and Toxic & classification of & the subst & nce) & & & & \\
\hline
\end{tabular}


TABLE 4: Brominated flame retardants (BFRs) declared in the list of 418 plastic additives actually used in the EU.

\begin{tabular}{|c|c|c|c|c|c|c|c|c|c|}
\hline Group and Substances & Name/Note & CAS No & Formula & $\begin{array}{l}\text { ECHA } \\
\text { (Clas- } \\
\text { sifica- } \\
\text { tion) }\end{array}$ & $\begin{array}{l}\text { ECHA } \\
\text { Notifica- } \\
\text { tions }\end{array}$ & BP & $\begin{array}{l}\text { Hazard Statement } \\
\text { Code, Concentration } \\
\text { Limit (Waste Hazard } \\
\text { Property) }\end{array}$ & $\begin{array}{l}\text { Low- } \\
\text { est } \\
\text { CL }\end{array}$ & $\begin{array}{l}\text { HP of } \\
\text { lowest } \\
\text { CL }\end{array}$ \\
\hline Hexabromocyclododecane & $\begin{array}{l}\text { Not in the scope } \\
\text { of this paper }\end{array}$ & $25637-99-4$ & & & $\begin{array}{l}\text { POP+oth- } \\
\text { ers }\end{array}$ & Yes & & & \\
\hline Bis(pentabromophenyl) ether & $\begin{array}{l}\text { Not in the scope } \\
\text { of this paper }\end{array}$ & $1163-19-5$ & & & $\begin{array}{l}\text { POP+oth- } \\
\text { ers }\end{array}$ & Yes & & & \\
\hline $\begin{array}{l}\text { 2,4,6-tris(2,4,6-tribromophe- } \\
\text { noxy)-1,3,5-triazine }\end{array}$ & $\begin{array}{l}\text { Group 3; in } \\
\text { catalog }\end{array}$ & $\begin{array}{l}426-040-2 \\
\text { (No CAS } \\
\text { number in } \\
\text { the file) }\end{array}$ & $\mathrm{C}_{21} \mathrm{H}_{6} \mathrm{Br}_{9} \mathrm{~N}_{3} \mathrm{O}_{3}$ & $\begin{array}{l}\text { No } \\
\text { HSC }\end{array}$ & PBT & Yes & & & \\
\hline $\begin{array}{l}\text { 1,1'-(ethane-1,2-diyl)bis[penta- } \\
\text { bromobenzene] }\end{array}$ & $\begin{array}{l}\text { Group 3; in } \\
\text { catalog }\end{array}$ & $84852-53-9$ & $\mathrm{C}_{14} \mathrm{H}_{4} \mathrm{Br}_{10}$ & $\begin{array}{l}\text { No } \\
\text { HSC }\end{array}$ & PBT & Yes & & & \\
\hline $\begin{array}{l}\mathrm{N}, \mathrm{N} \text { '-ethylenebis(3,4,5,6-tetra- } \\
\text { bromophthalimide) }\end{array}$ & $\begin{array}{l}\text { Group 3; in } \\
\text { catalog }\end{array}$ & $32588-76-4$ & $\mathrm{C}_{18} \mathrm{H}_{4} \mathrm{Br}_{8} \mathrm{~N}_{2} \mathrm{O}_{4}$ & $\begin{array}{l}\text { No } \\
\text { HSC }\end{array}$ & PBT & Yes & & & \\
\hline $\begin{array}{l}\text { 1,1'-(isopropylidene)bis[3,5-di- } \\
\text { bromo-4-(2,3-dibromoprop- } \\
\text { oxy)benzene] }\end{array}$ & $\begin{array}{l}\text { Group 3; in } \\
\text { catalog }\end{array}$ & $21850-44-2$ & $\mathrm{C}_{21} \mathrm{H}_{20} \mathrm{Br}_{8} \mathrm{O}_{2}$ & $\begin{array}{l}\text { No } \\
\text { HSC }\end{array}$ & PBT, ED & Yes & & & \\
\hline $\begin{array}{l}\text { 1,1'-(isopropylidene)bis[3,5-di- } \\
\text { bromo-4-(2,3-dibromo-2-meth- } \\
\text { ylpropoxy)benzene] }\end{array}$ & $\begin{array}{l}\text { Group 3; not in } \\
\text { catalog }\end{array}$ & 97416-84-7 & $\mathrm{C}_{23} \mathrm{H}_{24} \mathrm{Br}_{8} \mathrm{O}_{2}$ & $\begin{array}{l}\text { No } \\
\text { HSC }\end{array}$ & ED & Yes & & & \\
\hline $\begin{array}{l}\text { 2,2-bis(bromomethyl)pro- } \\
\text { pane-1,3-diol }\end{array}$ & $\begin{array}{l}\text { Group 3; not in } \\
\text { catalog }\end{array}$ & $3296-90-0$ & $\mathrm{C}_{23} \mathrm{H}_{24} \mathrm{Br}_{8} \mathrm{O}_{2}$ & $\begin{array}{l}\text { No } \\
\text { HSC }\end{array}$ & ED & Yes & & & \\
\hline $\begin{array}{l}\text { 2,2',6,6'-tetrabromo-4,4'-iso- } \\
\text { propylidenediphenol }\end{array}$ & $\begin{array}{l}\text { Group 4; not in } \\
\text { catalog }\end{array}$ & 79-94-7 & $\mathrm{C}_{15} \mathrm{H}_{12} \mathrm{Br}_{4} \mathrm{O}_{2}$ & $\begin{array}{l}\mathrm{H} 400 \\
\mathrm{H} 410\end{array}$ & PBT, ED & Yes & $\begin{array}{l}\mathrm{H} 400 \text { 25\% (HP 14) } \\
\text { H410 0.25\% (HP 14) }\end{array}$ & $0.25 \%$ & $\begin{array}{l}\text { HP } 14 \\
(\mathrm{H} 410)\end{array}$ \\
\hline
\end{tabular}

$B P=$ Brief Profile on ECHA site (a summary document on properties and classification of the substance)

ED list = "Under assessment as Endocrine Disrupting"

$H S C=$ hazard statement code

PBT list = "Under assessment as Persistent, Bioaccumulative and Toxic"

(HP 14 calculated without multiplying "M-factors")

The substances for which the dossier must be completed, or which are reassessed (according to the REACh regulation), are: the substances of 2 blends of Group 1, 7 substances ( 6 with ECHA notification) of Group 3, and 2 substances (with ECHA notification) of Group 4. Since there are mixtures, the total substances used that have to be assessed is 11 (or more depending on the composition of the 2 blends) out of 34 , of which 8 are notified by the ECHA.

The classification of product plastics incorporating these additives (according to the CLP) is beyond the scope of this study. For the simpler waste classification, the functional concentration of the additives in the plastics (recommended in the technical specifications) can be compared to the concentration that renders a waste hazardous (according to the waste regulations of EU 2014 and EU 2017).

In detail for the 12 classified substances (Table 3, Group 4), the number of substances, the concentration limits and the hazardous properties (for waste) are:

- 2 with $0.1 \%$ (for HP 7 'Carcinogenic' and HP 11 'Toxic for reproduction');

- 2 with $0.25 \%$ (HP 14 'Ecotoxic' chronic "high level");

- 1 with 10\% (HP 4 'Irritant' and HP 5 'Toxic');

with 20\% (HP 4 and / or HP 5), and

- 3 with 25\% (HP 14 'Ecotoxic' acute).

The two substances with a concentration limit of $0.1 \%$ are neopentyl tribromo alcohol [H350 0.1\% (HP 7), H340 0.1\% (HP 11), H341 1\% (HP 11)] and dibromo neopentyl glycol [H350 0.1\% (HP 7), H351 1\% (HP 7), H341 1\% (HP 11)]. The substances with a concentration limit of $0.25 \%$ are 2,4,6 tribromophenol and 2,2',6,6'-tetrabromo-4,4'-isopropylidenediphenol [0.25\% H410 (HP 14)]. According to their producer, the first three substances are used as a reactive intermediate for high molecular weight flame retardants, especially for rigid and flexible PU foam (the first two) and for the capping of brominated epoxides (the latter) (Table 5). These substances should normally not be found as separate substances in plastics but linked to the polymer matrix.

The 5 substances from the producer's catalog with ECHA notifications of assessment are presented in Table 6 with the information from their producer. Four substances are declared "additive" (they are miscible with the polymer but remain extractible and quantifiable) and they have 14 to 21 carbons. One substance (tribromophenol) is "reactive" (it will bind to the polymer and will not be found as a separate substance in a laboratory analysis) and has 6 carbons. Decabromodiphenylethane, the substitute of decabromodipheylether, present in the catalog of the three producers, and widely used today, is declared without hazard statement codes, but is under assessment in the PBT evaluation list of the ECHA.

To conclude, out of a total of 34 substances, 2 blends do not have a CAS number and dossier (Group 1), 7 substances have a CAS number and a dossier but are without any hazard statement (Group 3), among which 6 are under assessment by the ECHA, and 12 have a CAS number, a dossier and a hazard statement (Group 4), among which 
TABLE 5: Producer information on NR-BFRs with functional concentration greater than the concentration limit that renders a waste hazardous.

\begin{tabular}{|c|c|c|c|c|c|c|}
\hline Substance & Name & $\begin{array}{l}\text { Abbreviation / Brand } \\
\text { Name }\end{array}$ & Brand Name 2 - Note & CAS No & Formula & Category \\
\hline $\begin{array}{l}\text { Dibromo } \\
\text { neopentyl } \\
\text { glycol }\end{array}$ & & FR-522 & $\begin{array}{l}\text { FR- } 522 \text {, is a reactive, dibromoneopentyl glycol, flame retardant, } \\
\text { containing } 60 \% \text { aliphatic bromine. Thermosetting polyester } \\
\text { resins formulated with FR- } 522 \text { have high chemical and flame } \\
\text { resistance, minimal thermal discoloration and excellent light } \\
\text { stability. The high bromine content of FR-522 and its ready } \\
\text { reaction into polyurethanes make it suitable for use in rigid } \\
\text { polyurethane foams... }\end{array}$ & $\begin{array}{l}36483- \\
57-5\end{array}$ & $\begin{array}{l}\mathrm{C}_{5} \mathrm{H}- \\
{ }_{10} \mathrm{Br}_{2} \mathrm{O}_{2}\end{array}$ & Reactive \\
\hline $\begin{array}{l}\text { Tribromo } \\
\text { neopentyl } \\
\text { alcohol }\end{array}$ & $\begin{array}{l}\text { 2,2-di- } \\
\text { methylpro- } \\
\text { pan-1-ol, } \\
\text { tribromo } \\
\text { derivative }\end{array}$ & FR-513 & $\begin{array}{l}\text { FR- } 513 \text { is a reactive flame retardant containing } 73 \% \text { aliphatic } \\
\text { bromine. As tribromoneopentyl alcohol it combines a high bro- } \\
\text { mine content with exceptional stability, and is particularly suit- } \\
\text { able where thermal, hydrolytic and light stability are required. } \\
\text { FR- } 513 \text { high solubility in polyurethane systems increases } \\
\text { effectiveness as a reactive flame retardant for polyurethanes... }\end{array}$ & $\begin{array}{l}36483- \\
57-5\end{array}$ & $\mathrm{C}_{5} \mathrm{H}_{9} \mathrm{Br}_{3} \mathrm{O}$ & Reactive \\
\hline $\begin{array}{l}\text { 2,4,6-tribro- } \\
\text { mophenol }\end{array}$ & & FR-613 & $\begin{array}{l}\text { FR- } 613 \text { is a reactive flame retardant with a high content of } \\
\text { aromatic bromine, used mainly as an intermediate for high } \\
\text { molecular weight flame retardants. It is also an effective } \\
\text { fungicide and wood preservative... }\end{array}$ & $118-79-6$ & $\mathrm{C}_{6} \mathrm{H}_{3} \mathrm{Br}_{3} \mathrm{O}$ & Reactive \\
\hline
\end{tabular}

2 are under assessment by the ECHA. Eleven substances or more (due to the use of two blends) are re-assessed or could be re-evaluated in the future, and eventually restricted. The 7 non-POP NR-BFRs on the official list of plastic additives are currently being re-evaluated by the ECHA (3 PBT, 2 ED, 2 PBT + ED) (Table 4).

\section{CONCLUSIONS}

The regulated R-BFRs have been logically substituted by other non-regulated NR-BFRs. In this numerous data set, $88 \%$ of the product and waste samples contain BFR(s) other than the regulated ones.

The European Chemical Agency (ECHA) dossiers of these substances show that:

- 12 substances have no dossier (including polymers that must not be declared, and 3 substances not for sale in Europe);

- 10 substances have a dossier without hazard statements and are therefore not classified: The ECHA has, however, placed 6 of these substances on the PBT evaluation list ("under evaluation as persistent, bioaccumulative and toxic") and the ED evaluation list ("being assessed as an endocrine disruptor");

- 12 substances have a dossier and hazard statements (including 3 polymeric substances); however, the ECHA has placed 2 of these substances on the PBT evaluation list and ED evaluation list;

- The 3 substances that have a functional concentration (the concentration of additives that retards flame) greater than the concentration limits classifying a waste as hazardous, are declared as "reactive": they will bind to the polymer during their use and will not be independent substances anymore;

- The 7 non-POP NR-BFRs on the official list of plastic additives are currently being re-evaluated by ECHA (3 PBT, 2 ED, 2 PBT + ED).

As substitution is going faster than classification and regulation, and as homologous substances are used (for instance decabromodiphenylethane / decabromodipheny- lether), all these new NR-BFRs should be investigated and their properties on human health and the environment assessed and published, as already done for some, with the European Chemicals Agency (ECHA).

Total bromine is a relevant surrogate indicator of the presence of total BFR(s) in plastic waste, even if the brominated substances are not known and the new substances are currently not monitored for most samples. The technical limit of $2,000 \mathrm{mg} \mathrm{Br} / \mathrm{kg}$ as the maximum content for further recycling (EN 50625-3-1) takes into account all brominated substances regulated and not yet regulated, and therefore limits the recycling of BFRs that are not currently properly registered. Similar work should be done on phosphorus substitutes.

\section{ACKNOWLEDGMENTS}

This work has been supported by INERIS and by the Ministry for Ecologic Transition, France

\section{REFERENCES}

Albemarle 2018. Fire Safety Solutions - Product Selector Guide. https://www.albemarle.com/businesses/bromine-specialties/ fire-safety-solutions. Product information sheets consulted December 2020.

Beggio G, Hennebert P. 2021. Sampling and sub-sampling of granular waste: Part 2 - Size of a representative sample in terms of number of particles with different distributions of particle concentration and particle size. Submitted to Detritus.

Charbonnet J, Weber R, Blum A (2020) Flammability standards for furniture, building insulation and electronics: Benefit and risk. Emerging Contaminants 6, 432-441, https://doi.org/10.1016/j.emcon.2020.05.002

Chemtura Great Lakes Solution - Great Lakes Solution 2017. LANXESS Bromine Solutions. Flame retardants product guide. Consulted January 2018. Product information sheets consulted December 2020. http://add.lanxess.com/fileadmin/user_upload/Flame_Retardants_product_guide_04-2017.pdf

Chen SJ, Ma YJ, Wang J, Chen D, Luo XJ, Mai BX. Brominated flame retardants in children's toys: concentration, composition, and children's exposure and risk assessment. Environ Sci Technol. 2009 Jun 1;43(11):4200-6. doi: 10.1021/es9004834.

EN 50625-3-1:2015 Requirements for the collection, logistics and treatment of WEEE - Part 3-1: Specification relating to depollution - General. CENELEC, Brussels, Belgium. 
TABLE 6: Producer information on NR-BFRs with ECHA notifications.

\begin{tabular}{|c|c|c|c|c|c|c|}
\hline Substance & Abbreviation / Brand Name & Brand Name 2 - Note & CAS No & Formula & $\begin{array}{l}\text { ECHA Noti- } \\
\text { fications }\end{array}$ & Category \\
\hline $\begin{array}{l}\text { 2,4,6-Tris } \\
(2,4,6 \text {-tribromophe- } \\
\text { noxy) }-1,3,5 \text { triazine }\end{array}$ & FR-245 & $\begin{array}{l}\text { FR- } 245 \text { is a proprietary, additive } \\
\text { flame retardant. The combination } \\
\text { of aromatic bromine and cyanurate } \\
\text { provides high FR efficiency and } \\
\text { good thermal stability. A major use } \\
\text { of FR- } 245 \text { is in ABS and HIPS. FR- } \\
245 \text { combines good UV, impact and } \\
\text { flow properties... }\end{array}$ & $\begin{array}{l}25713- \\
60-4\end{array}$ & $\mathrm{C}_{21} \mathrm{H}_{6} \mathrm{Br}_{9} \mathrm{~N}_{3} \mathrm{O}_{3}$ & $\begin{array}{l}\text { PBT evalu- } \\
\text { ation list }\end{array}$ & Additive \\
\hline $\begin{array}{l}\text { Brominated flame } \\
\text { retardant, aromatic, } \\
\text { combined with an } \\
\text { imide structure }\end{array}$ & $\begin{array}{l}\text { SAYTEX® BT-93 Bromine-based } \\
\text { flame retardant for electronic enclo- } \\
\text { sures, electrical components, films, } \\
\text { and textiles that require the highest } \\
\text { degree of UV stability (light yellow } \\
\text { powder form). } \\
\text { SAYTEX® BT-93W Bromine-based } \\
\text { flame retardant for electronic enclo- } \\
\text { sures, electrical components, films, } \\
\text { and textiles that require outstand- } \\
\text { ing thermal and UV stability (white } \\
\text { powder form) }\end{array}$ & $\begin{array}{l}\text { SAYTEX BT-93 flame retardant is } \\
\text { a unique additive that combines } \\
\text { stable, aromatic bromine with an } \\
\text { imide structure. } \\
\text { SAYTEX BT-93W flame retardant } \\
\text { is a unique, white-colored additive } \\
\text { that combines stable, aromatic } \\
\text { bromine with an imide structure }\end{array}$ & $\begin{array}{l}32588- \\
76-4\end{array}$ & $\mathrm{C}_{18} \mathrm{H}_{4} \mathrm{Br}_{8} \mathrm{~N}_{2} \mathrm{O}_{4}$ & $\begin{array}{l}\text { PBT evalu- } \\
\text { ation list }\end{array}$ & Additive \\
\hline & Firemaster ${ }^{\circledR} 2100 \mathrm{R}$ & & & & & \\
\hline & $\begin{array}{l}\text { SAYTEX® } 8010 \text { Bromine-based } \\
\text { flame retardants for electronic } \\
\text { enclosures, electronic and electrical } \\
\text { components, insulation foams and } \\
\text { textiles (powder form) }\end{array}$ & $\begin{array}{l}\text { SAYTEX } 8010 \text { flame retardant is a } \\
\text { non-diphenyl oxide-based product } \\
\text { containing a high level of aromatic } \\
\text { bromine }\end{array}$ & & & & \\
\hline $\begin{array}{l}\text { Decabromodiphenyl } \\
\text { ethane }\end{array}$ & FR-1410 & $\begin{array}{l}\text { Decabromodiphenyl Ethane FR- } \\
1410 \text { is an additive flame retardant } \\
\text { containing } 82 \% \text { aromatic bromine. } \\
\text { Its high bromine content coupled } \\
\text { with its exceptional thermal stabil- } \\
\text { ity makes it the material of choice } \\
\text { for a large variety of applications. } \\
\text { Major application areas include } \\
\text { HIPS, Low and High Density Poly- } \\
\text { ethylene, Polypropylene (Homopoly- } \\
\text { mers and Copolymers), Elastomers, } \\
\text { PBT, Polyamides, UPE and Epoxy... }\end{array}$ & $\begin{array}{l}84852- \\
53-9\end{array}$ & $\mathrm{C}_{14} \mathrm{H}_{4} \mathrm{Br}_{10}$ & $\begin{array}{l}\text { PBT evalu- } \\
\text { ation list }\end{array}$ & Additive \\
\hline $\begin{array}{l}\text { 1,3-dibro- } \\
\text { mo-5-\{2-[3,5-dibro- } \\
\text { mo-4-(2,3-dibromo- } \\
\text { propoxy)phenyl] } \\
\text { propan-2-yl\}-2-(2,3-di- } \\
\text { bromopropoxy) } \\
\text { benzene }\end{array}$ & FR-720 & $\begin{array}{l}\text { FR-720 is an additive flame } \\
\text { retardant containing both aromatic } \\
\text { and aliphatic bromine. FR- } 720 \text { is } \\
\text { suitable for polyolefin and styrenic } \\
\text { resins and it is especially recom- } \\
\text { mended for UL- } 94 \text { class V- } 2 \text {. It is } \\
\text { also applicable in class V-0 polypro- } \\
\text { pylene. FR- } 720 \text { is cost efficient and } \\
\text { maintains good physical properties } \\
\text { of the compound... }\end{array}$ & $\begin{array}{l}21850- \\
44-2\end{array}$ & $\mathrm{C}_{21} \mathrm{H}_{20} \mathrm{Br}_{8} \mathrm{O}_{2}$ & $\begin{array}{l}\text { PBT } \\
\text { evaluation } \\
\text { list, ED } \\
\text { evaluation } \\
\text { list }\end{array}$ & Additive \\
\hline \multirow[t]{2}{*}{ 2,4,6-tribromophenol } & $\mathrm{PH}-73 \mathrm{FF}^{\mathrm{TM}}$ & $\begin{array}{l}\mathrm{PH}-73 \mathrm{FF}^{\mathrm{TM}} \text { is a reactive intermedi- } \\
\text { ate containing over } 70 \% \text { bromine } \\
\text { recommended for phenol-based } \\
\text { reactions in a free-flowing briquette } \\
\text { form. PH-73FF }{ }^{\mathrm{TM}} \text { by Lanxess is a } \\
\text { free flowing, non-caking, low dust- } \\
\text { ing, halogenated, flame retardant. It } \\
\text { is a reactive intermediate contain- } \\
\text { ing over } 70 \% \text { bromine recommend- } \\
\text { ed for phenol-based reactions } \\
\text { in a free-flowing briquette form. } \\
\text { PH-73FF }{ }^{\mathrm{TM}} \text { possesses uniform size } \\
\text { \& shape and high bulk density. It is } \\
\text { also used as an antifungal agent } \\
\text { (with FIFRA approval) or chemical } \\
\text { intermediate. }\end{array}$ & $118-79-6$ & \multirow[t]{2}{*}{$\mathrm{C}_{6} \mathrm{H}_{3} \mathrm{Br}_{3} \mathrm{O}$} & \multirow[t]{2}{*}{$\begin{array}{l}\text { PBT evalu- } \\
\text { ation list }\end{array}$} & \multirow[t]{2}{*}{ Reactive } \\
\hline & FR-613 & $\begin{array}{l}\text { FR- } 613 \text { is a reactive flame retardant } \\
\text { with a high content of aromatic bro- } \\
\text { mine, used mainly as an intermedi- } \\
\text { ate for high molecular weight flame } \\
\text { retardants. It is also an effective } \\
\text { fungicide and wood preservative. .. }\end{array}$ & & & & \\
\hline
\end{tabular}

PBT evaluation list: Under assessment as Persistent, Bioaccumulative and Toxic; ED evaluation list: Under assessment as Endocrine Disrupting 
EU 2014. Commission Decision 2014/955/EU of 18 December 2014 amending Decision 2000/532/EC on the list of waste pursuant to Directive 2008/98/EC of the European Parliament and of the Council.

EU 2017. Council Regulation (EU) 2017/997 of 8 June 2017 amending Annex III to Directive 2008/98/EC of the European Parliament and of the Council as regards the hazardous property HP 14 'Ecotoxic'. Official Journal of the European Union. 14.6.2017. L 150/1.

Fantke P, Weber R, Scheringer M (2015) From incremental to fundamental substitution in chemical alternatives assessment. Sustainable Chemistry and Pharmacy 1, 1-8. DOI: 10.1016/j.scp.2015.08.001

Haarman A, Magalini F, Courtois J. 2020. SOFIES. Study on the Impacts of Brominated Flame Retardants on the Recycling of WEEE plastics in Europe. SOFIES for BSEF. $44 \mathrm{p}$.

Hennebert P, Beggio G. 2021. Sampling and sub-sampling of granular waste: Part 1 - Size of a representative sample in terms of number of particles and application to waste containing rare particles in concentration. Submitted to Detritus.

Hennebert P. 2019. Sorting of waste for circular economy: sampling when (very) few particles have (very) high concentrations of contaminant or valuable element (with bi- or multi-modal distribution). 17th International Waste Management and Landfill Symposium (Sardinia 2019), 30/09 - 04/10/2019, Cagliari, Italy.
Hennebert P. 2020. Concentrations of brominated flame retardants in plastics of electrical and electronic equipment, vehicles, construction, textiles and non-food packaging: a review of occurrence and management. Detritus. DOI 10.31025/2611-4135/2020.13997

http://eurlex.europa.eu/legalcontent/EN/TXT/PDF/?uri=CELEX:32014D0955\&rid=1

ICL Industrial products. 2012. Fire protection for automotive and transportation. 6 p. http://icl-ip.com/wp-content/uploads/2012/10/ FR-Transportation-2012.pdf ; http://icl-ip.com/segment_category/ automotive/. Product information sheets consulted December 2020.

Lucas D, Petty SM, Keen O, Luedeka B, Schlummer M, Weber R, Barlaz $M$, Yazdani R, Riise B, Rhodes J, Nightingale D, Diamond ML, Vijgen J, Lindeman A, Blum A, Koshland CP (2018) Methods of Responsibly Managing End-of-Life Foams and Plastics Containing Flame Retardants: Part I Environmental Engineering Science 35 (6), 573587 https://doi.org/10.1089/ees.2017.0147

Wagner S, Schlummer M (2020) Legacy additives in a circular economy of plastics: Current dilemma, policy analysis, and emerging countermeasures. Resources, Conservation \& Recycling 158 (2020) 104800. https://doi.org/10.1016/j.resconrec.2020.104800 